\title{
An autopsy case of COVID-19 with a sudden death : Clinico-pathological comparison.
}

\author{
Yukiko Shishido-Hfara ${ }^{1}$, Keizo Furukawa ${ }^{2}$, Manabu Nishio ${ }^{2}$, Kohei Honda ${ }^{1}$, So Tando ${ }^{1}$, \\ Takeshi Yaoi ${ }^{1}$, Masataka Kawamoto ${ }^{1}$, Yosuke Maehara ${ }^{1}$, Takaaki Nakaya ${ }^{1}$, and Kyoko Itoh ${ }^{1}$ \\ ${ }^{1}$ Kyoto Prefectural University of Medicine \\ ${ }^{2}$ Kyoto Tanabe Central Hospital
}

March 5, 2022

\begin{abstract}
Autopsy was performed on a COVID-19 patient, who suddenly died despite the extensive anti-viral and anti-inflammatory therapies. Although moderate subpleural fibrosis was seen, pathology of DAD, a well-known cause for pulmonary failure, was minimum. Instead, severe hemorrhage was observed. Therapeutic effects were indicated, however why severe hemorrhage occurred was unclear.
\end{abstract}

\section{An autopsy case of COVID-19 with a sudden death : Clinico-pathological comparison.}

Yukiko Shishido-Hara ${ }^{*}$, Keizo Furukawa ${ }^{2}$, Manabu Nishio ${ }^{2}$, Kohei Honda ${ }^{3}$, So Tando ${ }^{1}$, Takeshi Yaoi $^{1}$, Masataka Kawamoto ${ }^{4,5}$, Yosuke Maehara ${ }^{6}$, Takaaki Nakaya ${ }^{5}$, and Kyoko Itoh $^{1}$

Department of Pathology and Applied Neurobiology

1. Department of Anatomic Pathology

2. Department of Forensics Medicine

3. Department of Infectious Diseases

4. Department of Radiology

Graduate School of Medical Science, Kyoto Prefectural University of Medicine, Kyoto, 602-8566, Japan.

Department of Cardiology, Kyoto Tanabe Central Hospital,

Kyotanabe city, Kyoto 610-0334, Japan

\section{Corresponding author:}

Yukiko Shishido-Hara, MD, PhD.

Department of Pathology and Applied Neurobiology

Graduate School of Medical Science, Kyoto Prefectural University of Medicine

465 Kajii-cho Kawaramachi-Hjirokoji, Kamigyo-ku, Kyoto 602-8566, Japan

shiyuki@koto.kpu-m.ac.jp Tel. and Fax: +81-75-251-5849

Written informed consent was obtained from the patient's next of kin to publish this report in accordance with the journal's patient consent policy

Running title: An autopsy case of COVID-19 
Figures: 6, Supplemental figures: 3

Key words: COVID-19, diffuse alveolar damage (DAD), SARS-CoV-2, subpleural fibrosis, thrombosis

\begin{abstract}
Autopsy was performed on a COVID-19 patient, who suddenly died despite the extensive anti-viral and antiinflammatory therapies. Although moderate subpleural fibrosis was seen, pathology of DAD, a well-known cause for pulmonary failure, was minimum. Instead, severe hemorrhage was observed. Therapeutic effects were indicated, however why severe hemorrhage occurred was unclear.
\end{abstract}

\title{
Introduction
}

Since December 2019, severe acute respiratory syndrome-coronavirus-2 (SARS-CoV-2) has been threatening the world. Despite the initial fears of this pandemic, an increasing number of autopsy cases have been reported, and pathophysiology of Coronavirus infectious disease 2019 (COVID-19) is getting better understood. Pathology of 662 patients in 58 studies from June 4, 2020 to September 30, 2020, was reviewed, and most frequently-described pulmonary findings were diffuse alveolar damage (DAD), followed by thromboembolisms, and non-specific shock injury in multiple organs ${ }^{1}$. Indeed, DAD, corresponding to the acute respiratory distress syndrome (ARDS) in the clinical status, is a major cause of death. Thus, therapeutic efforts have been made to save global lives.

Remdesivir is an RNA polymerase inhibitor, which has antiviral activities against RNA viruses ${ }^{2}$. The drug can incorporate into nascent viral RNA, and inhibit the RNA-dependent RNA polymerase, which results in premature termination of viral replication. Clinical course of COVID-19 is biphasic, consisting an early viral proliferating phase and a subsequent inflammatory phase ${ }^{3}$. Thus, remdesivir is effective with the first 5-day administration ${ }^{4}$. But later, depending on the severity of the patient's conditions, the optimal medication would differ.

Anti-inflammatory therapy is required, if the clinical condition worsened with overexuberant host immune response. After the anti-viral treatment, some patients may deteriorate, in approximately 7 days. Then, anti-inflammatory therapy starts to prevent unfavorable immune response, such as cytokine storm or ARDS. Here, glucocorticoids, such as dexamethasone, modulates inflammation-mediated lung injury and thereby is expected to reduce progression of respiratory failure ${ }^{5}$. Efficacy of dexamethasone was reported for hospitalized patients with respiratory support, either invasive mechanical ventilation or oxygen alone ${ }^{6}$. There is also growing interest in the use of tocilizumab, a monoclonal antibody against interleukin- 6 receptor (IL-6R). Although its efficacy is not yet concluded ${ }^{7}$, its additional use to corticosteroids has been reported beneficial ${ }^{8-10}$. Nonetheless, pathophysiological evidence is needed to prove therapeutic effects of these drugs.

Hear, we present an autopsy case of COVID-19 with treatment of remdesivir, dexamethasone, and tocilizumab. During the $4^{\text {th }}$ wave in Japan (April, 2021), biphasic anti-viral and anti-inflammatory treatment was applied with approval to overcome this disease. Unlike the previous autopsy reports conducted early in the pandemic, pathology reflecting the host unfavorable response, such as DAD and fibrosis was not so prominent in this case, likely suggesting the therapeutic effects. However, severe pulmonary hemorrhage seemed fatal, of which reason could not be concluded.

\section{Clinical summary}

A 75-year-old Japanese man was found to be infected with severe acute respiratory syndrome-coronavirus-2 (SARS-CoV-2) by polymerase chain reaction (PCR) test. He lived together with his wife and daughter, and his daughter first developed Coronavirus infectious disease 2019 (COVID-19). Three days later, he and his wife appeared to be PCR-positive for the virus. He was under medication for diabetes mellitus $(\mathrm{DM})$, gout, hypertension, and atrial fibrillation. In addition, he underwent an operation for squamous cell carcinoma, two months before the PCR test. His status was pT2aN2M0, pStageIIIa, and he showed a good post-operative performance. 
Three days after the PCR test, he developed a fever, and was hospitalized (day 1). His $\mathrm{SpO}_{2}$ was $95 \%$ with oxygen, $2 \mathrm{~L} / \mathrm{min}$ by nasal canula. The image of thoracic computed tomography $(\mathrm{CT})$ is presented in Figure 1a. Anti-viral therapy with remdesivir $(100 \mathrm{mg} /$ day $)$ was started, but his fever did not go down. On day 5, CT revealed the apparent pulmonary fibrosis with a crazy-paving pattern, characteristic for COVID-19. Oxygen was supplied with nasal high frow (NHF), 40L, $\mathrm{FiO}_{2} 55 \%$. Anti-inflammatory therapy was started with dexamethasone $\left(6.6 \mathrm{mg}\right.$ /day). However, with worsened respiratory condition, $\mathrm{FiO}_{2} 70-80 \%$ was required to keep $\mathrm{SpO}_{2}$ 90-95\%. Invasive mechanical ventilation was seriously considered, but not performed due to unwillingness of the patient and his family. On day 10, IL-6 inhibitor, tocilizumab (680mg) was administered, then $\mathrm{SpO} 2$ was gradually improved. Since $\mathrm{FiO}_{2}$ was decreased to $40-60 \%$ to keep $\mathrm{SpO}_{2} 92-95 \%$, NHF was changed to the biphasic positive airway pressure system (BIPAP). However, on day 15, bacterial co-infection was suspected with the white blood cell (WBC) count, 23600/ $\mu$ l. Increase in $\mathrm{FiO}_{2}$ was again required. and treatment with meropenem (2g/day) was started. Pulse therapy with prednisolone (1000 $\mathrm{mg} \times 3$ days) was also done. On day 21, he was suddenly deteriorated with bradycardia and low blood pressure, and passed away. Just before the autopsy, the final PCR test still revealed the presence of SARS-CoV-2 in the nasopharyngeal swab. The clinical course is summarized in Figure 1.

\section{Pathological findings}

Autopsy was performed 8 hours 20 min after his death. The height $177 \mathrm{~cm}$ and weight $92 \mathrm{~kg}$ indicated obesity (BMI 29.4). Operation scar for lung cancer was present in the left lateral side of his chest, as well as multiple insulin-injection scars in his abdomen.

\section{Pulmonary pathology}

Macroscopically, both lungs were hardened, weighed $800 \mathrm{~g}$ (right upper, middle, and lower lobes) and $260 \mathrm{~g}$ (left lower lobe)(Figure 2a). The right lung was markedly hemorrhagic, especially in the middle lobe. The upper lobe of the left lung was missing, due to lobectomy for lung cancer. The cut surfaces of post-mortem lungs were compared with the last CT image taken on day 8 (Figure 2b)(Supplement figures 1-3)

Severe pulmonary hemorrhage in the right lung

The right lung was markedly hemorrhagic, especially in the hilar region of the middle lobe. The hemorrhagic lesion was extended to both upper and lower lobes (Figure 3). In the last computed radiography (CR) images taken on day 17 , four days before the death, hemorrhagic signs were not observed, indicating the hemorrhage occurred during the last three days. The right upper lobe was affected with emphysema, and the lower lobe, subpleural fibrosis. Blood filled most healthy alveoli, indicating the fatality (Figure 4a). The bronchi in the right lower lobe were mildly enlarged, in which coagulants were present (Figure 5ab).

Thrombosis in the pleural and subpleural regions.

Thrombosis was observed in the pulmonary veins of the thickened pleura and small vessels in the subpleural area (Figure $4 \mathrm{ab}, 5 \mathrm{~d}$ ). In the right middle lobe, old thrombus was organized and recanalized (Figure 4b, read arrow). However, most thrombi were fresh without organization (Figure 4ab, 5d). Hemorrhagic area included necrotic alveoli and blood vessels, as well as fragmented alveolar septum, where the presence or absence of thrombi was hardly evaluated. The cause of pulmonary hemorrhage was not be concluded. Although hemorrhagic infarct was one of the possibilities, it usually occurs in the subpleural area, which is distinct from this case.

Peri-lobular fibrosis in the subpleural area

Subpleural fibrosis was predominantly seen in in the lateral-to-dorsal side of the bilateral lower lobes. Pleura was fibrously thickened, and fibrosis was extended along with lobular septum, making peri-lobular patterns (Figure 5ad). Masson trichrome (MT) staining revealed that the fibrosis was apparent in the band-like area (width 3-4 mm) of the subpleural region (Figure 5d).

Other pulmonary pathology 
The DAD pathology in the exudative phase, hyaline membrane formation inside of the alveolar lumen, was seen, but focal (Figure 4c). The end-stage pathology of DAD was also present in the hemorrhagic lesion, accompanying squamous metaplasia (Figure 5c). Although previous autopsy reports described the DAD pathology is the major cause of lung failure ${ }^{1,11}$, it was minimum in the present case. Infiltration of neutrophils and lymphocytes were also hardly seen, and only macrophages predominantly appeared. Immunohistochemistry in use of anti-SARS-CoV-2 antibody did not reveal any infected cells. Although PCR showed the viral RNA presence in the nasopharyngeal swab, the discrepancy would be due to the detection sensitivity. Thus, even if the virus was present, its copy number would be low.

\section{Systematic pathology of other organs}

COVID-19 is a systematic disease, in which multiple organs, including the lungs, heart, liver, kidney, gastrointestinal tract, the central nervous system (CNS) would be affected ${ }^{12,13}$. Full autopsy of the present case revealed varieties of findings in other organs, but it was uncertain if they are directly associated with COVID-19, since bacterial and fungus co-infection was also observed. Additional pathological findings are described as follows.

Stomach: Severe hemorrhage with multiple ulcers. Candida infection was detected.

Heart: Diffuse neutrophil infiltration in the myocardium and stromal edema. Mural thrombus in the left auricular appendage.

Spleen: Acute splenitis with prominent neutrophil infiltration.

Kidney: Multiple abscess formation with bacterial colonies. Microcoagulopathy in glomerular blood vessels, resembling disseminated intravascular coagulation (DIC).

Lymph nodes: Lymph adenitis and erythrophagocytosis.

Prostate: Microabscess.

Liver: Mild lymphocytic infiltration in the portal area

Again, hemorrhage was severe in the right lung, as well as stomach. The clinical data of PT-INR, APTT, PT, D-dimer and platelet was reviewed, but the cause of severe hemorrhage still remained uncertain (Figure $6)$.

\section{Discussion}

In this report, we present an autopsy case of COVID-19 with anti-viral and anti-inflammatory therapy. Pulmonary pathology revealed severe hemorrhagic change, but the findings of DAD and subpleural fibrosis were not so prominent. Autopsy findings of COVID-19 have been reported from worldwide countries, including the United States ${ }^{13}$, and those from Europe ${ }^{14-16}$, Asia ${ }^{12,17,18}$, and Africa ${ }^{19}$. No national differences have been described, and most reports stated that DAD is a major cause of respiratory failure, which is different from the present case.

Since this pandemic occurred, efforts have been made to overcome the exaggerated host immune reactions, and a living WHO guideline on drugs for COVID-19, for example, was repeatedly updated ${ }^{20}$. Autopsy of the present case was performed in April, 2021 during the $4^{\text {th }}$ pandemic wave in Japan, and the patient was treated with anti-inflammatory therapy with dexamethasone and tocilizumab. Thus, the absence of active DAD and severe fibrosis would be due to the therapeutic effects. Subpleural fibrosis was also restricted. Thus, we interpret that the direct cause of death was severe hemorrhage, but not DAD.

The reason why severe hemorrhage occurred remain undetermined. In the kidney, microvascular thrombosis was seen in the glomerular vessels, resembling pathology of DIC, although the last platelet count was over $90,000 / \mu \mathrm{l}$ in the peripheral blood. The patient was also under anticoagulation therapy with warfarin, due to permanent atrial fibrillation. His PT-INR was kept between 1.8-3.8 in the last day. At the autopsy, his stomach was filled with blood, and multiple ulceration was seen accompanying candida colonies. In kidney, 
bacterial colonies were also present with multiple abscess. Thus, co-infection may relate to the systematic coagulation and fibrinolysis disorder, but it could not be concluded.

In conclusion, we present the autopsy case of COVID-19 treated with remdesivir, dexamethasone, and tocilizumab. The pulmonary pathology suggested the therapeutic effect, but the cause of severe hemorrhage remain unknown. Co-infection would be also a future issue in relation to anti-inflammatory therapy for COVID-19. We hope our analysis contributes to save the global lives from this pandemic disease.

\section{Acknowledgement}

We thank Takashi Okuda and Toshifumi Kawamura for excellent technical assistance, and Syoko Kirito and Keisuke Nishioka for virological assistance.

\section{Ethics statement}

The investigation was conducted in accordance with the Declaration of Helsinki of 1975, and approval by an institutional ethics committee is not required due to the nature of case report.

\section{Disclosure statement}

None declared.

\section{Author contribution}

YS-H and KH prepared the manuscript and figures. YS-H and ST performed autopsy. TY, MK and TN performed molecular detection of the virus. YM analyzed the CT images. KF and MN cared the patient and prepared clinical data. KI designated this study.

References1 Caramaschi S, Kapp ME, Miller SE, et al. Histopathological findings and clinicopathologic correlation in COVID-19: a systematic review. Mod Pathol. 2021; 34: 1614-33. 2 Agostini ML, Andres EL, Sims AC, et al. Coronavirus Susceptibility to the Antiviral Remdesivir (GS-5734) Is Mediated by the Viral Polymerase and the Proofreading Exoribonuclease. mBio. 2018; 9. 3 Cevik M, Kuppalli K, Kindrachuk J, Peiris M. Virology, transmission, and pathogenesis of SARS-CoV-2. BMJ. 2020; 371: m3862. 4 Goldman JD, Lye DCB, Hui DS, et al. Remdesivir for 5 or 10 Days in Patients with Severe Covid-19. N Engl J Med. 2020;383: 1827-37. 5 Group RC, Horby P, Lim WS, et al. Dexamethasone in Hospitalized Patients with Covid-19. $N$ Engl J Med. 2021; 384: 693-704. 6 Gandhi RT. The Multidimensional Challenge of Treating COVID-19: Remdesivir is a Foot in the Door. Clin Infect Dis. 2020. 7 Stone JH, Frigault MJ, Serling-Boyd NJ, et al. Efficacy of Tocilizumab in Patients Hospitalized with Covid-19. N Engl J Med. 2020; 383: 2333-44. 8 Group RC. Tocilizumab in patients admitted to hospital with COVID-19 (RECOVERY): a randomised, controlled, open-label, platform trial.Lancet. 2021; 397: 1637-45. 9 Brown MJ, Alazawi W, Kanoni S. Interleukin-6 Receptor Antagonists in Critically Ill Patients with Covid-19. N Engl J Med. 2021;385: 1147. 10 Gordon AC, Angus DC, Derde LPG. Interleukin-6 Receptor Antagonists in Critically Ill Patients with Covid-19. Reply. N Engl J Med. 2021;385: 1147-49. 11 Schaller T, Hirschbuhl K, Burkhardt $\mathrm{K}$, et al. Postmortem Examination of Patients With COVID-19. JAMA. 2020; 323: 2518-20. 12 Yao XH, Luo T, Shi Y, et al. A cohort autopsy study defines COVID-19 systemic pathogenesis. Cell Res. 2021; 31: 836-46. 13 Bryce C, Grimes Z, Pujadas E, et al. Pathophysiology of SARS-CoV-2: the Mount Sinai COVID-19 autopsy experience. Mod Pathol. 2021; 34: 1456-67. 14 Calabrese F, Pezzuto F, Fortarezza F, et al. Pulmonary pathology and COVID-19: lessons from autopsy. The experience of European Pulmonary Pathologists. Virchows Arch. 2020; 477: 359-72. 15 von Weyhern CH, Kaufmann I, Neff F, Kremer M. Early evidence of pronounced brain involvement in fatal COVID-19 outcomes. Lancet. 2020; 395: e109. 16 Falasca L, Nardacci R, Colombo D, et al. Postmortem Findings in Italian Patients With COVID-19: A Descriptive Full Autopsy Study of Cases With and Without Comorbidities. J Infect Dis. 2020;222: 1807-15. 17 Adachi T, Chong JM, Nakajima N, et al. Clinicopathologic and Immunohistochemical Findings from Autopsy of Patient with COVID-19, Japan. Emerg Infect Dis. 2020; 26. 18 Okudela K, Hayashi H, Yoshimura Y, et al. A Japanese case of COVID-19: An autopsy report. Pathol Int. 2020; 70: 820-24. 19 Keshinro SO, Awolola NA, Adebayo LA, Mutiu WB, Saka BA, Abdus-Salam IA. Full autopsy in a confirmed 
COVID-19 patient in Lagos, Nigeria - A case report. Hum Pathol (N Y). 2021; 24: 200524. 20 Update to living WHO guideline on drugs for covid-19. BMJ. 2021; 374: n2219.

\section{Figure Legends}

Figure 1

(a) Representative CT images. Progression of subpleural fibrosis was apparent on day 5, but on day 8 . (b) Oxygen dosage and medication during the 21-day clinical course. (c) SpO2 improved temporarily after administration of tocilizumab. (d) The summary of WBC, CRP and body temperature. On day 15, the WBC count and body temperature increased, followed by CRP.

\section{Figure 2}

(a) Gross appearance of postmortem lungs. The left upper lobe is missing after lobectomy for cancer. (b) After formalin fixation, the right lung was sectioned into A-Q. Sections I, K, and L roughly corresponded to the slices $\mathrm{A}, \mathrm{B}$, and $\mathrm{C}$ in the $\mathrm{CR}$ images.

\section{Figure 3}

The CT images on day 8 were compared with the postmortem lung. Slices A, B, and C on CT roughly corresponded to the sections I, K, and L, respectively. The upper lobe Emphysema in the upper lobe in the lower lobe were recognized. Marked hemorrhage was seen in the right middle lobe of the postmortem lung, which was absent in CT images. Red arrows in section I and section L correspond to the histopathology shown in Figure 4a and 5a, respectively.

\section{Figure 4}

Histopathology of the right upper and middle lobes (right arrow in Figure 3a). (a) Emphysema and thrombosis were observed in the upper lobe, while severe hemorrhage in the middle lobe. (b)High power view of thrombosis (red arrow in a). Organized thrombus is pointed with the red arrow. (c) Hight power view of the middle lobe (blue arrow in a). DAD with fibrin exudation was focally observed. (a): Masson trichrome (MT) staining. (b)(c): Hematoxylin-eosin (HE) staining.

\section{Figure 5}

Histopathology of the right lower lobe (red arrow in Figure 3c).(a) Perilobular fibrosis was seen, in the subpleural area, width 3-4mm. (b) High power view of intrabronchial coagulants (red arrow in a). (c) High power view of squamous metaplasia (blue arrow in a). (d) High power view of the subpleural fibrosis (black arrow in a).

\section{Figure 6}

Clinical dynamics of the coagulation system and the dose of warfarin. (a) PT-INR was the highest on day 8, but improved with vitamin K supplementation and reduction of warfarin. (b) PT and APTT. After day 8, APTT was prolonged over PT, suggesting APTT dominance.(c) D-dimer was the highest on day 8, but declined during the latter part of the clinical course. (d) Platelet count in the peripheral blood was the lowest on day 17 , but readily recovered since then.

\section{Supplementary materials}

Supplementary figure 1: Macroscopic findings of the right lung, and semi-macroscopic observations of sections $\mathrm{C}$ and I.

Supplementary figure 2: Semi-macroscopic observations of sections of J and L.

Supplementary figure 3:

Macroscopic findings of the left lung, and semi-macroscopic observation of sections L. 
(a)

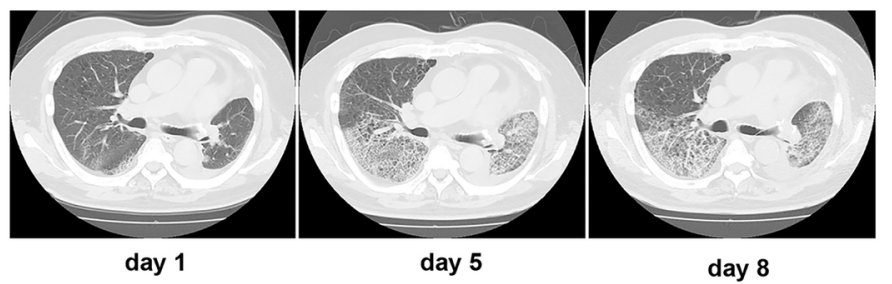

(b)

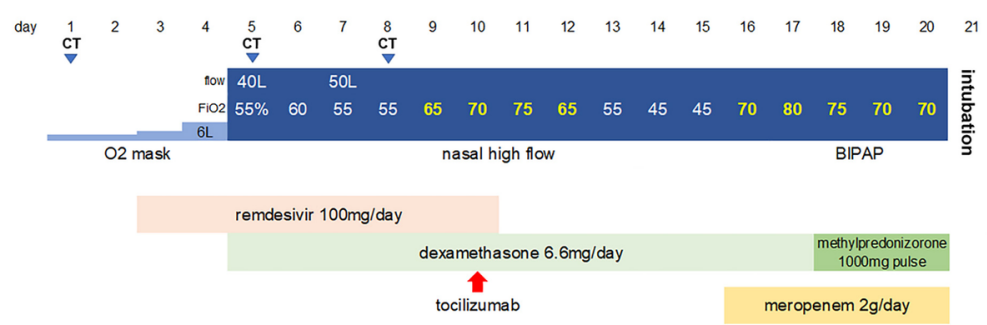

(c)

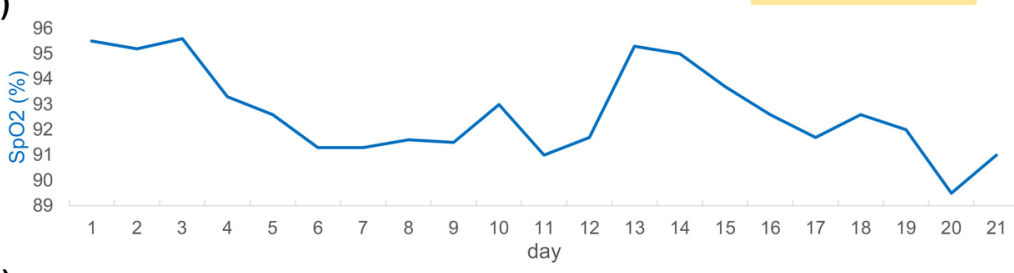

(d)

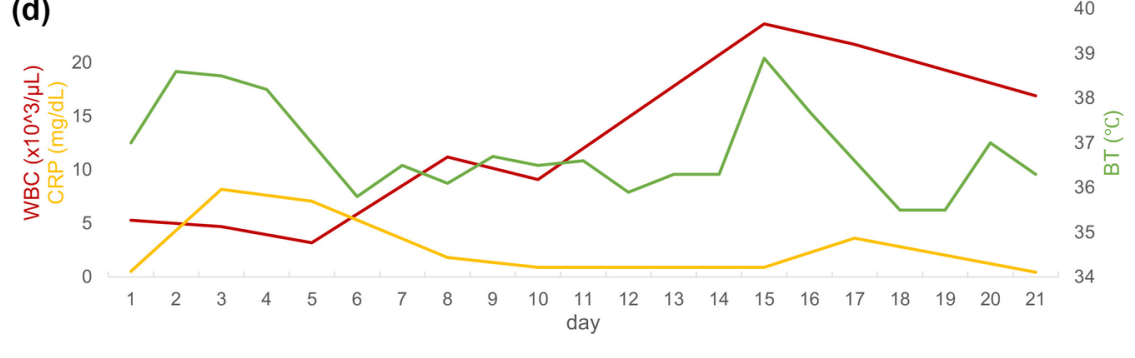


(a)
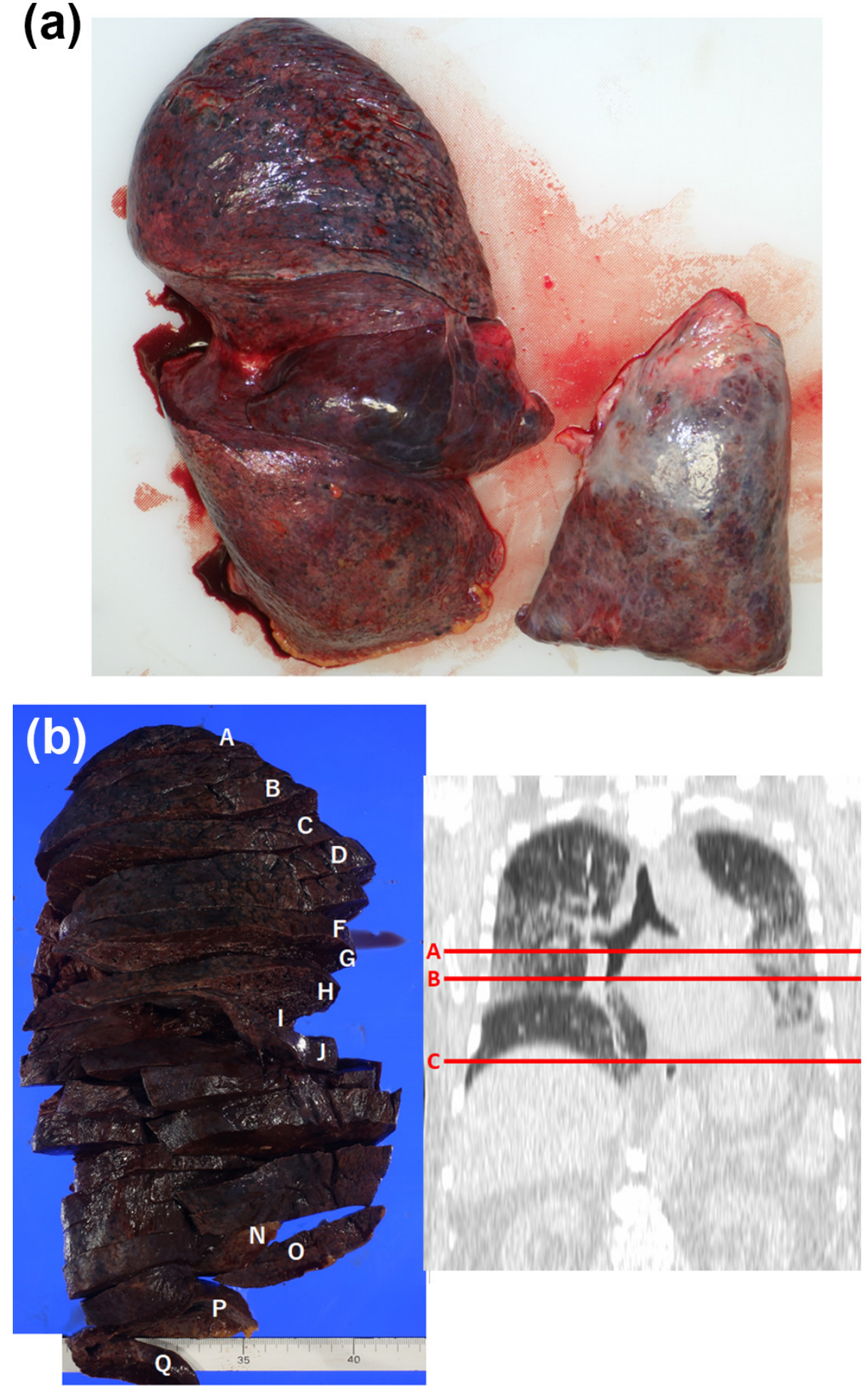
(a) Slice A

(b) Slice B

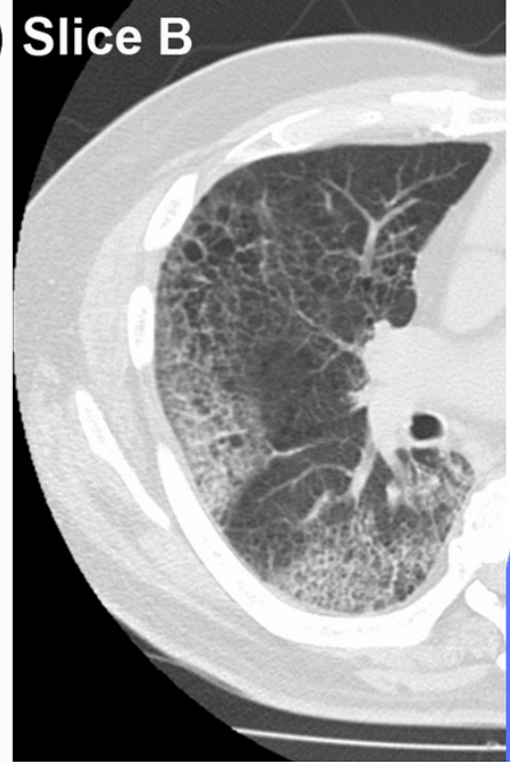

(c)

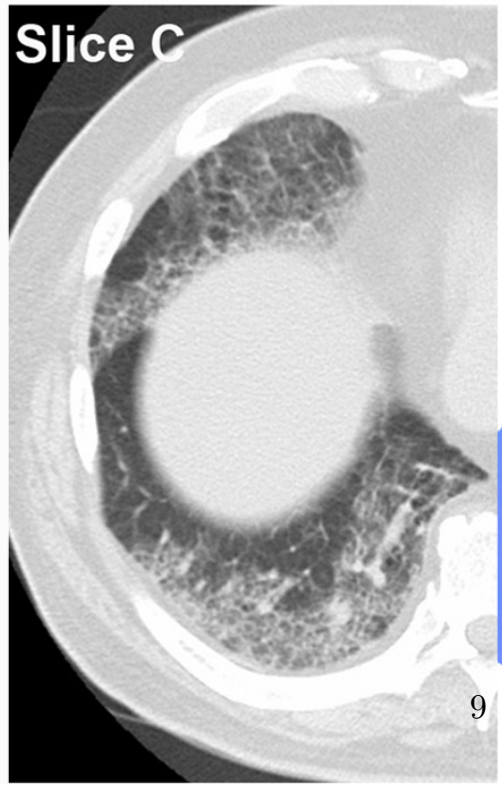

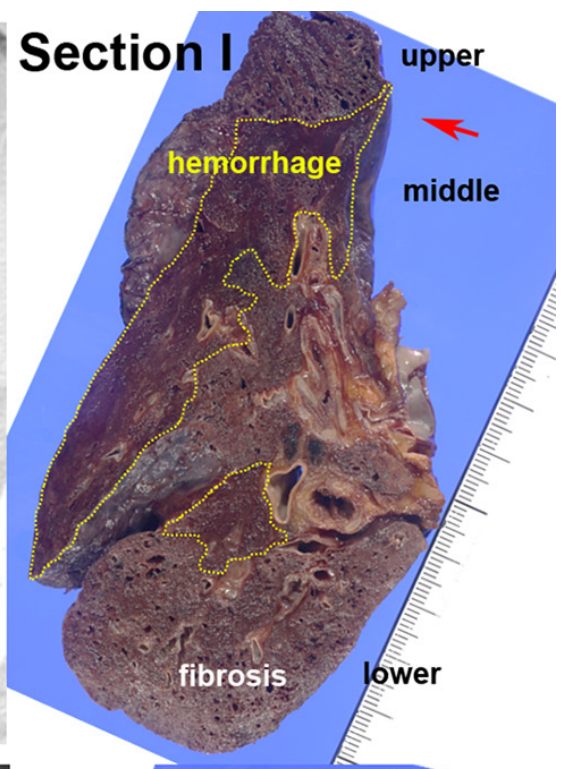

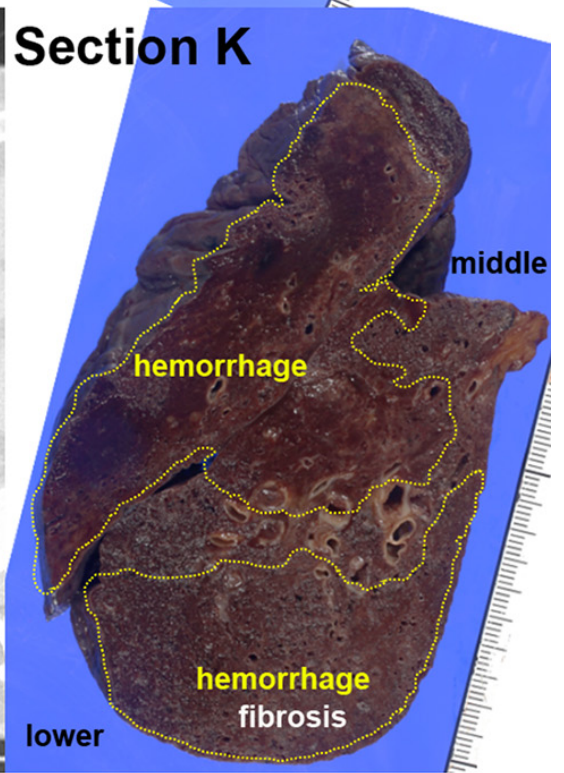

Section L

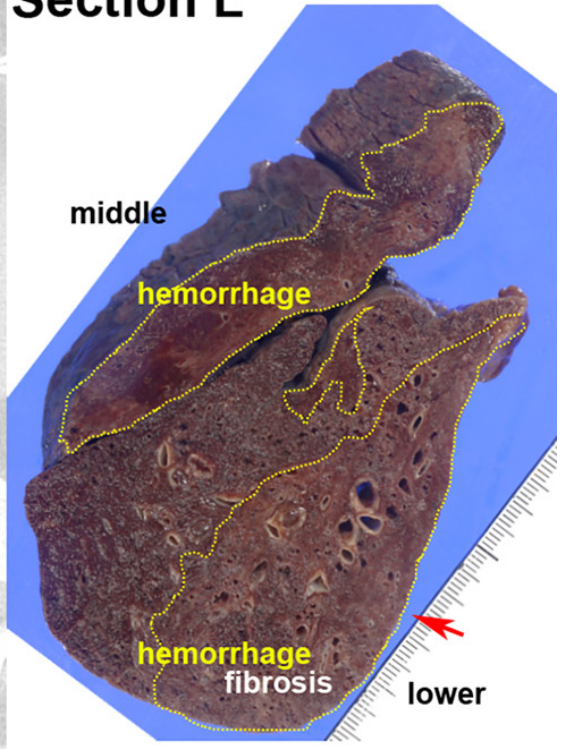



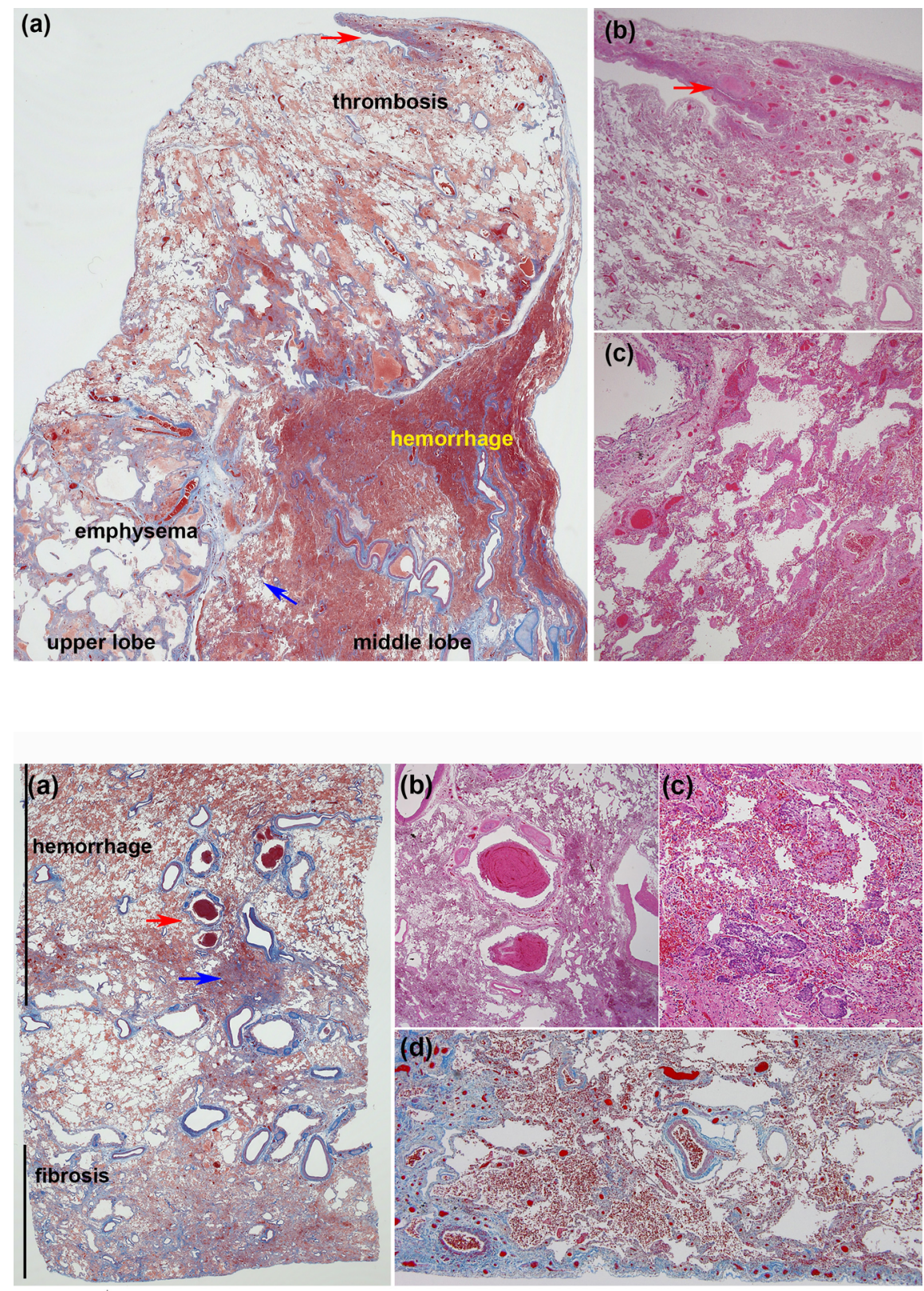

$\uparrow$ 
(a)

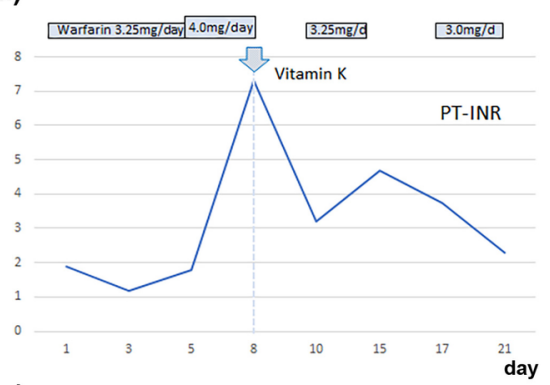

(c)

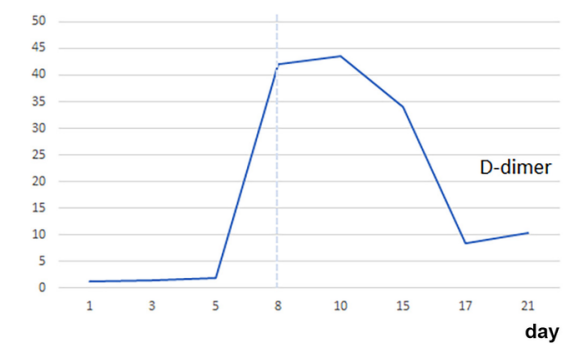

(b)

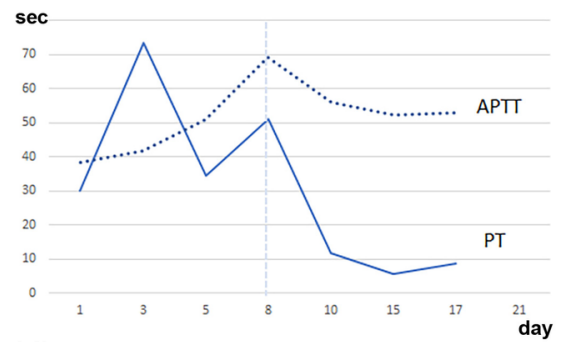

(d)

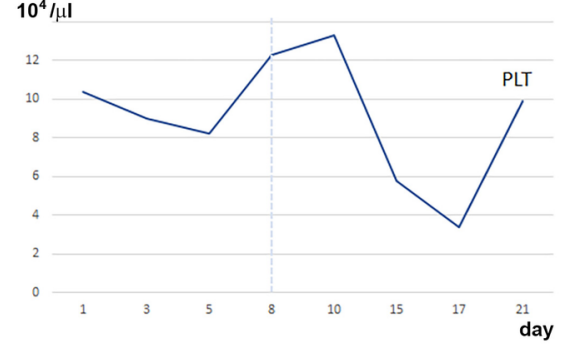

